\title{
FENÓTIPO PERIODONTAL: UMA VISÃO CLÍNICA E ATUAL
}

\author{
PERIODONTAL PHENOTYPE:A CLINICAL AND CURRENT VIEW
}

\author{
Lucas Mendes Gabri', Victor Gila Gomes de Mattos', \\ Luis Paulo Diniz Barreto², Marcela Melo dos Santos ${ }^{2}$
}

\section{Resumo}

Um dos elementos essenciais para alcançar a estética do sorriso é o fenótipo e o contorno gengival que, com suas arquiteturas, influenciam no tamanho das coroas dentais. $\bigcirc$ termo "fenótipo periodontal" foi padronizado no Workshop Mundial para a Classificação das Doenças e Condições Periodontais e Peri-Implantares de 2017 , porém esse tema já havia sido abordado outras vezes, com outras nomenclaturas. A avaliação dos diferentes fenótipos periodontais é fundamental, pois nos dão informações relacionadas às características dos tecidos periodontais e às formas dentárias, além de tornar o tratamento mais previsível, podendo evitar problemas como: trauma, inflamação e outras complicações clínicas e cirúrgicas. $\bigcirc$ objetivo desta revisão de literatura é fazer uma pesquisa a respeito do tema fenótipo periodontal por meio dos artigos mais relevantes entre o período de 2017 a 2021, evidenciando sua classificação, prevalência e formas de diagnóstico. Existem várias formas de diagnosticar o fenótipo periodontal, sendo a transparência do sulco gengival por meio da sonda milimetrada a preconizada pelo Workshop Mundial. Podemos observar uma prevalência do fenótipo fino pelo gênero feminino, e o fenótipo espesso pelo gênero masculino. Ainda faltam mais evidências científicas para o correto relacionamento do fenótipo periodontal com outros fatores como idade, tabagismo, hábitos de higiene, alimentação e má oclusão.

Palavras-chave: Periodontia, Gengiva, Fenótipo.

\begin{abstract}
One of the essential elements to achieving smile esthetics is the phenotype and gingival contour, which with their architecture influence the size of dental crowns. The term "periodontal phenotype" was standardized in the 2017 World Workshop on the Classification of Periodontal and PeriImplant Diseases and Conditions. However, much has been said about the topic, with other nomenclatures. Evaluating different periodontal phenotypes is essential, as they provide us with information related to the characteristics of periodontal tissues and dental forms. In addition to making the treatment more predictable, it can avoid problems such as trauma, inflammation, and other clinical and surgical complications. The purpose of this literature review is to research the topic, periodontal phenotype, through the most relevant articles between the period 2017 to 2021, showing its classification, prevalence, and forms of diagnosis. There are several ways to diagnose the periodontal phenotype, and the one recommended by the World Workshop is the transparency of the gingival sulcus using the millimeter probe. We can observe a prevalence of the thin phenotype for the female gender, whereas the thick phenotype is prevalent for the male gender. There is still a lack of scientific evidence for the correct relationship of the periodontal phenotype with other factors such as age, smoking, hygiene habits, diet and, malocclusion.
\end{abstract}

Keywords: Periodontics, Gums, Phenotype.

I. Graduando em Odontologia. Universidade do Grande Rio, Rio de Janeiro, Brasil.

2. Professor Mestre do Departamento de Periodontia da Universidade do Grande Rio, Rio de Janeiro, Brasil.

Como citar este artigo:

Gabri LM, Mattos VGG, Barreto LPD, Santos MM. Fenótipo periodontal: uma visão clínica e atual. Rev Nav Odontol. 202 I; 48(2): .26-36. 


\section{INTRODUÇÃO}

A busca pela estética é algo muito visado atualmente, estando ligada a regras e princípios da arte (I). Os elementos essenciais para alcançar a estética do sorriso são o fenótipo e o contorno gengival, que, com sua arquitetura, influencia no tamanho das coroas dentais $(2,3)$. A avaliação do fenótipo periodontal é fundamental na clínica diária do cirurgião-dentista, principalmente durante o planejamento de procedimentos em áreas estéticas do paciente. Sendo assim, sua identificação é relevante para um bom prognóstico, evitando trauma, inflamação e outras complicações clínicas e cirúrgicas (4).

Em 2017, o Workshop Mundial para a Classificação das Doenças e Condições Periodontais e Peri-Implantares recomendou a adoção do termo "fenótipo periodontal", que é descrito como a combinação do fenótipo gengival e sua morfologia óssea. Fenótipo gengival é constituído pela espessura gengival, pela sua dimensão de gengiva queratinizada e pela espessura de sua tábua óssea vestibular. Fenótipo indica uma dimensão que pode mudar de acordo com o tempo, dependendo de fatores ambientais e intervenções clínicas, ou seja, o fenótipo pode ser modificado (5).

Existem diversas classificações desses fenótipos gengivais na literatura, sendo a mais usual a que diferencia entre fino e espesso. $\bigcirc$ fenótipo gengival fino possui pouca espessura, com isso, sua resposta para inflamações e traumas é mais suscetível a recessões. Por sua vez, fenótipo espesso apresenta uma gengiva mais fibrosa e, na presença de uma reação inflamatória ou após um trauma, pode ocorrer a formação de bolsa periodontal (6). Durante um planejamento que vise a manter a estética do paciente, o cirurgião-dentista também deve ter atenção à anatomia da arcada dentária do paciente, como o seu formato e suas proporções. Um fenótipo periodontal fino apresenta coroas triangulares, com uma arquitetura óssea afilada e gengiva fina e delicada. Já o fenótipo do tipo espesso apresenta coroas dentárias mais retangulares e um processo alveolar mais robusto e uma gengiva espessa (7).

Para chegar ao diagnóstico, alguns métodos são relatados na literatura, dentre eles: medição direta da espessura gengival utilizando limas endodônticas com cursores, determinação ultrassônica, avaliações de transparência gengival com uma sonda milimetrada, realização de tomografias computadorizadas por feixe cônico com afastadores labiais e tomadas radiográficas bidimensionais para avaliar a espessura óssea, scanner a laser, scanners CAD/CAM e fotografia intraoral $(8,9)$.

O diagnóstico do fenótipo periodontal é fundamental para o correto planejamento do tratamento odontológico. $\bigcirc$ correto diagnóstico pode afetar os resultados do tratamento devido às diferentes formas de resposta inflamatória que cada tecido gengival possui (6). Tendo isso em mente, o profissional pode ter uma melhor previsibilidade de como o tecido periodontal irá reagir durante e após o ato cirúrgico ou terapêutico, evitando quaisquer defeitos estéticos (7). O objetivo desta revisão de literatura é fazer uma pesquisa a respeito do tema fenótipo periodontal por meio dos artigos mais relevantes entre o período de 2017 a 2021 , evidenciando sua classificação, prevalência e formas de diagnóstico.

\section{REVISÃO DA LITERATURA}

\section{Histórico das classificações}

Nos anos 2000, surgiram novos trabalhos que investigavam os diferentes fenótipos periodontais, além da proposição de um novo método simples e antes nunca empregado, o método visual baseado na transparência da sonda periodontal através da margem gengival $(|\mathrm{O}, \mathrm{I}| \mathrm{I})$.

Muller et al. realizaram um estudo para comprovar os dados obtidos em seus estudos anteriores e identificaram três grupos denominados $A I, A 2$ e $B(12,13)$. Sendo Al: espessura gengival fina, faixa de tecido queratinizado estreita, dentes mais alongados; A2: espessura gengival fina, ampla faixa de tecido queratinizado, dentes mais alongados; B: espessura gengival grossa, ampla faixa de tecido queratinizado, dentes de formato mais quadrado (12)

O início da utilização das tomografias computadorizadas de cone beam (TCCB) trouxe a oportunidade de obter uma visualização mais clara e medições mais precisas das estruturas periodontais e, assim, oportunidades de futuros estudos sobre a análise do fenótipo periodontal (I4). 
De Rouck et al. confirmaram a existência dos três grupos ( $A$ I , A2, B), antes propostos por Muller et al., em um estudo usando o método visual por transparência de sondagem $(12,15)$. Segundo eles, o grupo AI parecia corresponder a um fenótipo "festonado", enquanto o grupo B possuía características de um biótipo "plano espesso". Porém, não havia classificação possível para o grupo A2 (15). Eghbali et al. usaram a mesma análise de De Rouck et al. para avaliar a precisão da inspeção visual direta como um método para diagnosticar o fenótipo gengival $(|5| 6$,$) . Finalmente, o fenótipo espesso foi sub-$ classificado em "festonado espesso" e "plano espesso", assim considerando que aquele fenótipo "festonado" teria características iguais às dos outros fenótipos mais extremos $(15,16)$.

Posteriormente, estudos investigaram a confiabilidade dos métodos de transparência por sondagem e TCB (tomografia de tecido mole) para diagnóstico do biótipo gengival, espessura clínica da gengiva vestibular e do osso $(17,18)$.

Os dados obtidos em estudos anteriores mostram que os três fenótipos periodontais ("festonado fino", "festonado espesso" e "espesso") são diagnosticados com base em características como espessura gengival, morfologia gengival, morfologia óssea e dimensões dentárias (19). Outros dados relatam que o fenótipo fino está associado a uma espessura mais fina da tábua óssea vestibular, diferentemente do fenótipo periodontal espesso (20).

Nos anos entre 20 I I e 20 l 8, principalmente no ano de 2017, no qual ocorreu o Workshop Mundial sobre a classificação de doenças e condições periodontais e peri-implantares, houve um grande desenvolvimento nos estudos sobre saúde e estética periodontais. Com o aumento de pesquisas relacionadas ao desenvolvimento das recessões gengivais associadas ao fenótipo gengival, o grupo de trabalho de Kim et al. destacou as diferenças entre biótipo e fenótipo (2l). Termo usado anteriormente, biótipo é algo geneticamente predeterminado e não pode ser modificado por fatores ambientais ou cirúrgicos (5). Já o fenótipo pode ser modificado com base na combinação de características genéticas e fatores ambientais, ou seja, uma interação entre genótipo e o ambiente (22). $\bigcirc$ recente Workshop Mundial recomendou a adoção do novo termo fenótipo para descre- ver a combinação do fenótipo gengival, que envolve a espessura gengival, largura da gengiva queratinizada e morfologia óssea, ou seja, sua espessura (5). Além da regularização do novo termo, também foi sugerido utilizar uma sonda periodontal de forma padronizada e reprodutível para medir a espessura gengival, avaliando o instrumento a partir de sua transparência após inserido no sulco (5).

Na Tabela I, observa-se em resumo a metodologia e os resultados de estudos realizados entre os anos de 1997 a 2020. Assim, explicitando a evolução da classificação dos fenótipos periodontais com o passar dos anos.

\section{Classificação}

As características do fenótipo gengival estão relacionadas diretamente com o processo alveolar e a morfologia dos dentes, eventos que ocorrem durante erupção dentária e com a inclinação e a posição dos dentes erupcionados (23).

$\bigcirc$ fenótipo gengival espesso possui uma arquitetura óssea densa e gengiva fibrosa, podendo ser facilmente observado clinicamente, acompanhando uma topografia gengival plana (Figura I). Este possui uma quantidade ampla de gengiva inserida. Dessa forma, sua resposta à inflamação é mais favorável, tendendo à formação de bolsas periodontais e proporcionando uma melhor previsibilidade a procedimentos cirúrgi$\cos (4)$. Portadores de uma gengiva espessa frequentemente apresentam coroas com formato quadrado e convexidade acentuada, além disso a papila interproximal é pequena e o ponto de contato localiza-se mais apicalmente (23).

$\bigcirc$ fenótipo gengival fino é mais delicado e translúcido, possuindo uma arquitetura óssea mais fina, geralmente acompanhada de fenestrações e deiscências ósseas (Figura 2) (4). Por ser um tecido mais sensível, sua resposta à inflamação é mais severa, podendo ocorrer recessões gengivais. Tal fenótipo é geralmente acompanhado de dentes longos e finos, com convexidade leve e formato triangular (23). Na tabela 2, podemos observar as características de cada fenótipo segundo Kao e Pasquinelli (4).

Um estudo de Amid et al. mostrou que a idade média dos indivíduos com diferentes fenótipos gengivais ao redor dos incisivos cen- 


\section{AUTORES}

Harris RJ, 1997

(I0)

Muller et al., $2000(12)$

\section{METODOLOGIA}

Método visual pela transparência por sondagem.

Avaliaram todos os dentes pelo método ultrassônico e paquímetro.

De Rouck et al., 2009 (I5)

Eghbali et al., 2009 (16)

Kan et al., 2010 (I7)

Fu et al., 2010 (I8)

Cook et al., 20II (20)

Zweers et al., 2014 (19)

Jepsen et al., $2018(5)$

Kim et al., 2020 (2I)
Avaliaram incisivos maxilares pelo método da transparência por sondagem.

Utilizaram slides apresentando fotografias clínicas de dentes anteriores na região de maxila.

Utilizaram método de transparência por sondagem, e paquímetros com tensão reajustada.

Avaliaram dentes da região anterior de maxila em cadáveres, utilizando método de transparência por sondagem, $\mathrm{TCB}$, paquímetros com tensão reajustada.

Avaliaram dentes da região anterior de maxila pelos métodos de transparência por sondagem, $\mathrm{TCB}$, scanners.

Reuniram uma série de artigos relevantes sobre as várias formas de fenótipo periodontal e seus métodos de diagnóstico.

Reuniram uma série de estudos sobre doenças periodontais e peri-implantares.

Reuniram uma série de artigos a partir de três questões sobre o papel do fenótipo periodontal na manutenção da saúde periodontal.

\section{RESULTADOS}

Fenótipo fino foi classificado quando sua espessura fosse menor que $0.5 \mathrm{~mm}$, sendo visível pela transparência. Fenótipo espesso foi classificado quando não era possível visualizar a sonda pela transparência no sulco.

Grupo Al: espessura gengival fina, faixa de tecido queratinizado estreita, dentes mais alongados; A2: espessura gengival fina, ampla faixa de tecido queratinizado, dentes mais alongados; $\mathrm{B}$ : espessura gengival grossa, ampla faixa de tecido queratinizado, dentes de formato mais quadrado.

Fenótipo festonado referente ao grupo AI (fino); Fenótipo plano espesso referente ao grupo B (espesso).

Fenótipo gengival "espesso-festonado"; fenótipo "espesso-plano"; fenótipo "fino-festonado".

Fenótipo fino, sendo caracterizado como uma gengiva fina e friável, com transparência na sondagem e espessura gengival menor que $1.0 \mathrm{~mm}$. Fenótipo espesso, caracterizado por uma gengiva fibrótica, sem transparência na sondagem, espessura gengival maior que $1.0 \mathrm{~mm}$.

Fenótipo fino, com transparência visível na sondagem. Fenótipo espesso, sem transparência visível na sondagem.

Fenótipo “espesso/médio" quando não há transparência por sondagem, está associado a uma tábua óssea vestibular mais espessa.

Fenótipo "fino" quando há transparência pela sondagem, possui tábua óssea mais afilada.

A partir dos dados obtidos nos estudos avaliados, os três fenótipos periodontais são diagnosticados com base em características como espessura gengival, morfologia gengival, morfologia óssea e dimensões dentárias.

Realizaram uma mudança no termo antes conhecido por "biótipo periodontal" para "fenótipo periodontal". O termo consiste no conjunto de "fenótipo gengival","morfologia óssea" e largura de gengiva queratinizada.

A partir dos dados obtidos, percebeu-se que indivíduos com fenótipo periodontal fino eram mais suscetíveis a recessões gengivais quando submetidos a algum trauma ou cirurgias. 


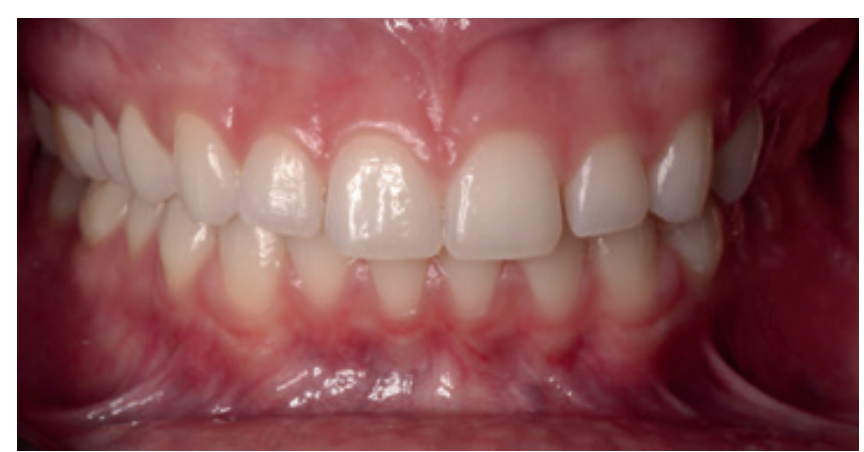

Figura I - Fenótipo periodontal espesso

Fonte: Imagem cedida gentilmente pelo professor Luis Paulo Barreto e pela professora Marcela Melo.

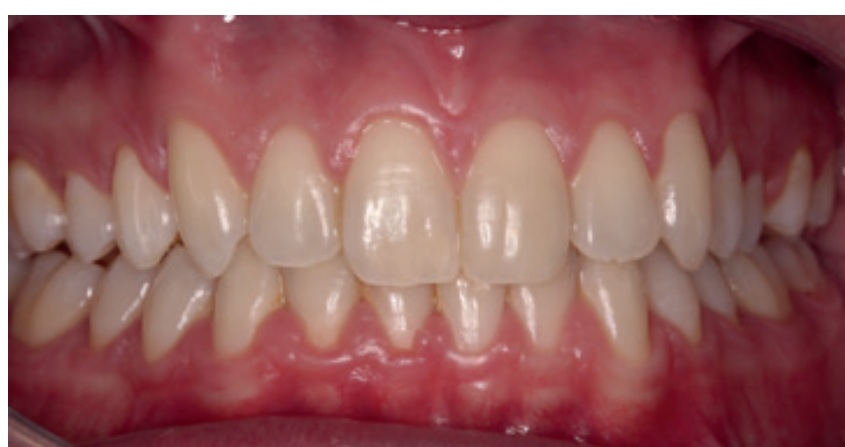

Figura 2 - Fenótipo periodontal fino

Fonte: Imagem cedida gentilmente pelo professor Luis Paulo Barreto e pela professora Marcela Melo.

Tabela 2 - Características dos Fenótipos Fino e Espesso (Kao e Pasquinelli, 2002)

\begin{tabular}{|c|c|}
\hline FENÓTIPO FINO & FENÓTIPO ESPESSO \\
\hline Tecido mole fino. & Tecido mole denso e fibrótico. \\
\hline Estreita faixa de tecido queratinizado. & Larga faixa de tecido queratinizado. \\
\hline $\begin{array}{c}\text { Menos de } 1,5 \mathrm{~mm} \text { de espessura e } \\
\text { largura entre } 3,5 \text { e } 5 \mathrm{~mm} .\end{array}$ & $\begin{array}{c}\text { mm ou mais de espessura gengival } \\
\text { e largura entre } 5 \text { e } 6 \mathrm{~mm} .\end{array}$ \\
\hline $\begin{array}{c}\text { Tecido duro festonado e fino (Deiscências e } \\
\text { fenestrações são achados habituais). }\end{array}$ & Tecido duro plano e espesso. \\
\hline Osso marginal fino. & Osso marginal espesso. \\
\hline Áreas de contato no terço incisal/ oclusal. & Área de contato no terço médio/ cervical. \\
\hline Coroas triangulares. & Coroas quadrangulares. \\
\hline Papilas longas e estreitas. & Papilas curtas e largas. \\
\hline
\end{tabular}

Fonte: Kao e Pasquinelli, 2002 (4)

trais, laterais e caninos não foi significantemente diferente (24). A média do desvio padrão da espessura gengival em $2 \mathrm{~mm}$ apical à margem gengival foi de 1,35-0,29 mm para incisivo central, I,23-0,29 mm para laterais e I, I 5-0,27 $\mathrm{mm}$ para caninos. Esses valores foram significantemente diferentes entre os dentes, e a espessura gengival média dos incisivos centrais foi maior do que os laterais e caninos. Além disso, esse valor dos laterais foi maior que nos caninos. A porcentagem de frequência de fenótipos finos e espessos nos dentes anteriores superiores não mostrou parâmetros diferentes entre homens e mulheres. A porcentagem de frequência de fenótipos gengivais foi diferente entre caninos e incisivos centrais, e também entre as áreas centrais e incisivos laterais (24).

Em uma pesquisa do ano de 2015 , foi feita uma amostra de 55 adultos (24 homens e 31 mulheres) com idades entre 18 e 35 anos (25). Os participantes apresentavam placa vi- sível, sangramento gengival e sangramento à sondagem de aproximadamente 15\% a 20\%. Dos dentes avaliados, 11,5\% exibiam perda de inserção, e com relação à recessão gengival foi obtida uma média de 1,01 mm. A espessura gengival média foi de I a $1,97 \mathrm{~mm}$, tendo como conclusão a correlação entre espessura gengival e recessão gengival. Sendo assim, quanto menor a espessura gengival, maior será a recessão gengival (25).

Posteriormente, no estudo de Karakis et al., em 2019, foi feito um acompanhamento de 6 meses em um grupo de 31 pacientes que apresentava pouca largura de gengiva queratinizada, em que foi submetido à cirurgia de enxerto gengival livre (26). Foi utilizado para determinar o fenótipo gengival o método da transparência da sonda periodontal. Os pacientes foram divididos em 2 grupos, de acordo com seu fenótipo gengival (fino e espesso), e foram realizadas as medidas de alguns parâ- 
metros clínicos. Como resultado, não houve diferenças estatisticamente significativas nas medidas clínicas e cirúrgicas entre os grupos (26).

Em um estudo mais recente de Yuan et al., um total de 40 jovens voluntários com periodonto saudável foram envolvidos nessa pesquisa (27). Por meio da sonda periodontal, foi determinado o fenótipo gengival em espesso e fino. Dois registros foram medidos por tomografia computadorizada de cone beam (TCCB): a espessura gengival (GT) foi medida no nível da junção cemento-esmalte; e a espessura óssea foi medida em 3 locais — I, 3 e 5 mm abaixo da crista alveolar. Medidas orais e em modelos de gesso foram utilizadas para analisar as associações da relação largura da coroa / comprimento da coroa (CW / CL), largura da mucosa queratinizada (KM) e curvatura da margem gengival livre. Os jovens que apresentavam fenótipo espesso tiveram a espessura gengival $\geq 1 \mathrm{~mm}$, já os com fenótipo fino tiveram a espessura gengival $<1 \mathrm{~mm}$. Os resultados mostram uma associação entre espessura óssea com GT, CW / CL, KM e curvatura da margem gengival livre (27).

Yin et al. elaboraram um estudo para avaliar a correlação entre o fenótipo gengival na área estética e perfil craniofacial, baseando-se em tomografias computadorizadas de feixe cônico (28). Foram ao total 56 participantes (I3 homens e 43 mulheres), com a idade média de 23,6 anos. Os maxilares foram escaneados usando um scanner intraoral, e nesses modelos foram realizados, para descrição da relação posicional entre a dentição da maxila e os pontos de referência gengivais, um plano de referência horizontal baseado no plano oclusal, com 22 marcos. Os resultados mostraram que o fenótipo espesso representou a maior proporção (69,6\%) dos 56 participantes do estudo (28).

\section{Formas de diagnóstico}

A sonda periodontal é um instrumento obrigatório para o cirurgião-dentista, tendo um papel indispensável para o diagnóstico clínico periodontal do paciente. Tal instrumento é usado para diversos exames, como profundidade de bolsa, nível de inserção clínica, sangramento à sondagem, largura da gengiva inserida e para avaliar o espaço biológico (8). A sonda milimetrada também pode ser usada para diagnosticar o fenótipo periodontal, sendo esse um dos métodos mais empregados e menos invasivos. A técnica consiste em posicionar a sonda periodontal no sulco vestibular em um dente e caso a sonda possa ser vista através da gengiva, o fenótipo gengival é considerado fino; seguindo a mesma lógica, caso a sonda não seja vista, o fenótipo é considerado espesso (8).

Workshop de 2017 recomenda utilizar a sonda periodontal de forma padronizada e reprodutível para medir a espessura gengival,

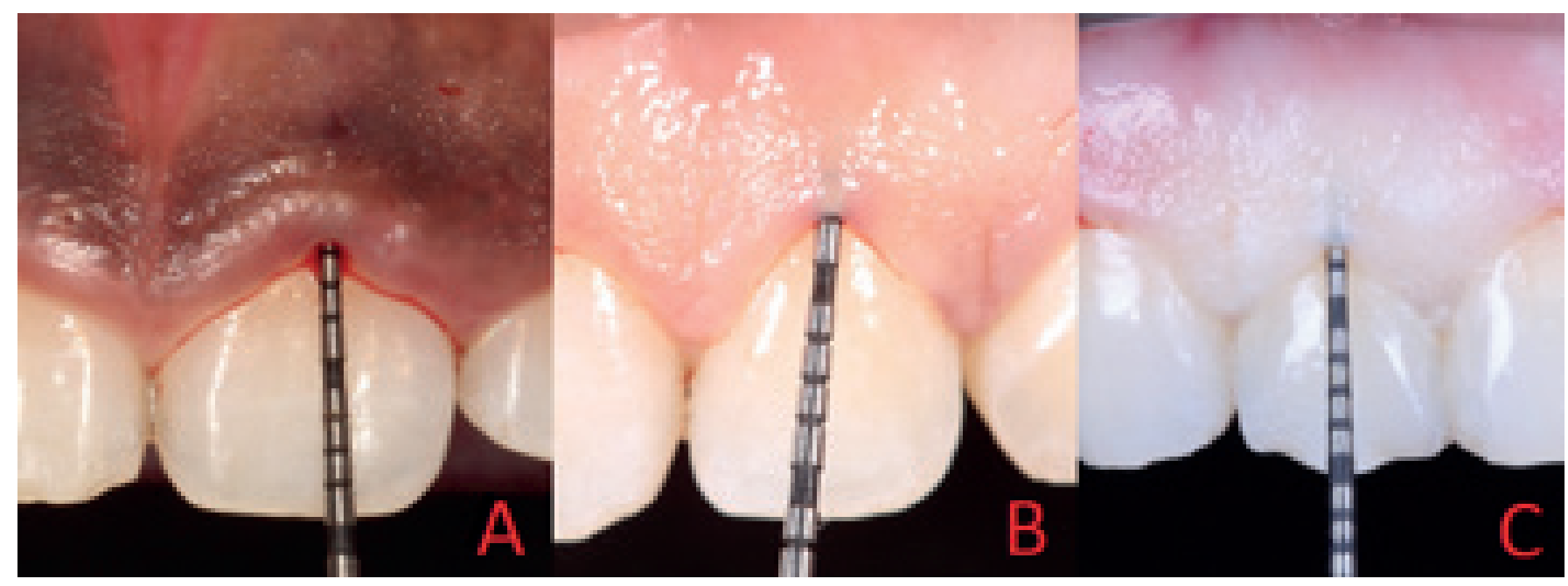

Figura 3 - Diagnóstico do fenótipo periodontal por meio da técnica da sondagem. A foto A representa o fenótipo espesso, a foto $B$ representa o fenótipo intermediário e a foto $C$ o fenótipo fino. Fonte: Imagem cedida gentilmente pelo professor Luis Paulo Barreto e pela professora Marcela Melo 

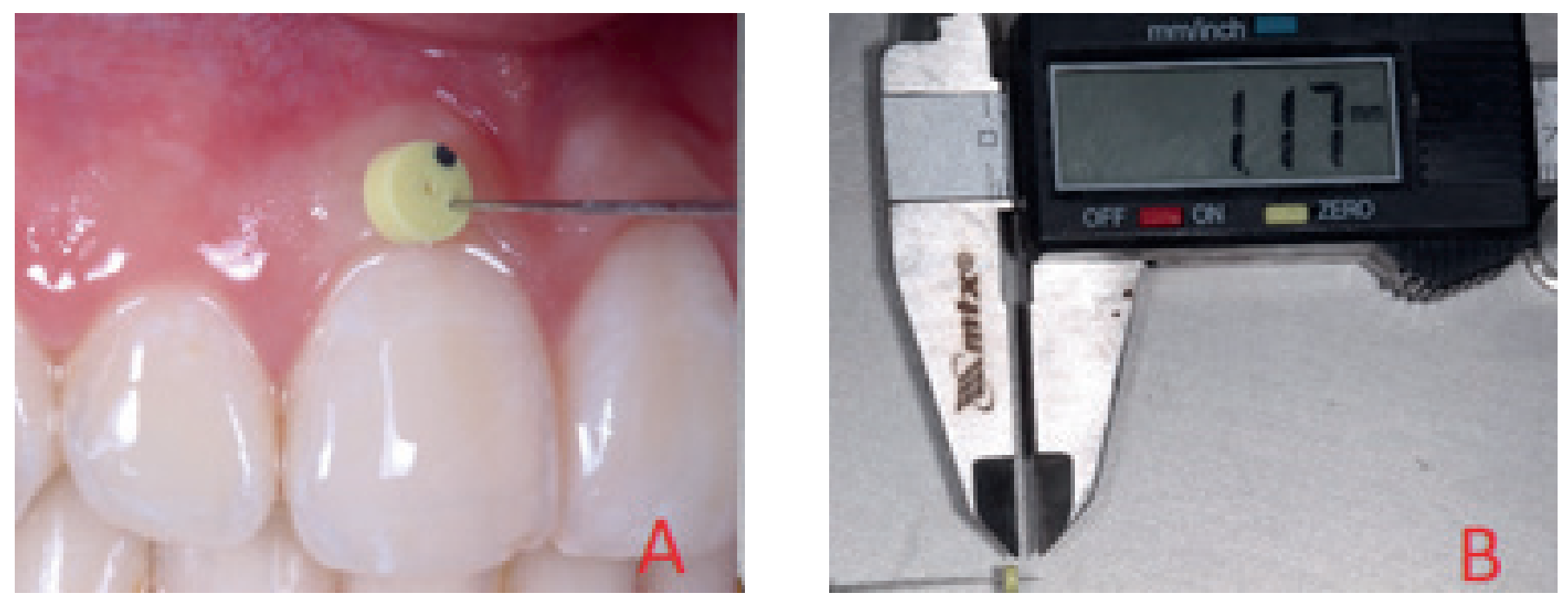

Figura 4 - Na foto $A$ é realizado o diagnóstico do fenótipo periodontal com uso de um espaçador digital para medir a espessura gengival. Já na foto $B$ é medida a espessura gengival com um paquímetro digital.

Fonte: Imagem cedida gentilmente pelo professor Luis Paulo Barreto e pela professora Marcela Melo.

avaliando o instrumento a partir de sua transparência após inserido no sulco (5).

A tomografia computadorizada de feixe cônico, utilizando afastadores labiais, é um exame que possibilita uma visão tridimensional das estruturas anatômicas. As medidas radiográficas do tecido duro e mole, assemelham-se com as clínicas, deixando o diagnóstico previsível e preciso (18). Em 2018, um estudo mostrou que o método de TC para classificar a espessura e a largura do tecido gengival apresenta valores aceitáveis de confiabilidade e pode ser considerado útil clinicamente, principalmente para classificar fenótipo periodontal espesso (9).

método transgengival (Figura 4) é realizado utilizando uma lima endodôntica número 15 com stop de borracha, inserindo-a no centro entre a margem gengival e a junção mucogengival de forma perpendicular. A medição é registrada com uma sonda periodontal, ou com um paquímetro digital (29). Previamente ao procedimento, deve-se anestesiar a gengiva com anestésico tópico para evitar desconforto do paciente. $\bigcirc$ método permite uma boa medição da espessura gengival, porém, como é invasivo, a anestesia local pode atrapalhar, pois aumenta o volume e pode gerar um desconforto durante as medições (8).

Segundo um estudo realizado por Muller et al., o dispositivo ultrassônico produz informações válidas e relativamente confiáveis sobre a espessura da maioria das partes da mucosa mastigatória da cavidade oral (30). É um método pouco invasivo e tem um bom custo-benefício. É um dispositivo que realiza impulsos através do tecido gengival que refletem na superfície do osso e do dente. Esses impulsos retornam ao dispositivo, que exibe os resultados digitalmente (30).

A fotografia intraoral é um dos métodos menos invasivos, porém é necessário que o profissional tenha conhecimento das características e prevalência dos fenótipos periodontais, além de uma experiência clínica para o correto diagnóstico. Em 2018, Araújo et al. enviaram questionários para profissionais formados, com o objetivo de avaliar a capacitação profissional em diagnosticar o fenótipo gengival de 3 fotografias distintas (3I). Como conclusão, foi visto que os dentistas formados há mais de 10 anos tiveram facilidade na correlação correta dos fenótipos, enquanto os profissionais com menos anos de formados tiveram mais facilidade em identificar o fenótipo espesso e tiveram dificuldade de diferenciar o fenótipo fino do intermediário. Ressalta-se, porém, que a dificuldade persiste até para os especialistas em periodontia e prótese dentária (3I).

Houve um estudo, realizado em 2018 por Alves et al., com objetivo de avaliar as propriedades dos métodos de avaliação dos fenótipos gengivais (9). Foram incluídos indivíduos de ambos os sexos, de 18 a 65 anos, já programados 
para realizar tomografias computadorizadas. Todos deveriam ter periodonto saudável, ausência de cáries, recessões gengivais, próteses, aparelhos ortodônticos, e não fazer uso de medicamentos. Grávidas e fumantes também foram excluídos. Os dentes anteriores da maxila de 12 indivíduos foram submetidos à avaliação da espessura gengival pelos métodos: transparência por sondagem, transgengival, fotográfico e tomografia computadorizada de cone beam (TCCB). Foram submetidos a esse estudo 7 homens e 5 mulheres com idades entre 24 e 35 anos, tendo suas espessuras médias de 1,44 $\mathrm{mm}$ pelo método transgengival, I,37 $\mathrm{mm}$ pela TCCB. A maior concordância entre os valores obtidos foi encontrada nos métodos transgengival e TCCB. Além disso, em consenso entre os 3 avaliadores, a confiabilidade do método fotográfico era ruim. O método de TCCB para classificar a espessura e largura do tecido gengival apresenta valores aceitáveis de confiabilidade e pode ser considerado útil clinicamente, principalmente para classificar fenótipo espesso (9).

Em um estudo de 2020, realizado por Gkogkos et al., foi avaliada a espessura gengival dos incisivos inferiores centrais de 20 cadáveres suínos, utilizando diversos métodos de diagnóstico (32). Foi comparada a avaliação por meio de duas formas do método transgengival, uma utilizando uma sonda periodontal e a outra por uma agulha de acupuntura. Os resultados foram quase idênticos (espessura gengival média I, I | mm vs. I, I 4 mm para o incisivo esquerdo e espessura gengival I, I2 mm vs. I, I I mm para o incisivo direito, respectivamente). Outra comparação foi entre a avaliação pelo ultrassom e pela tomografia computadorizada de cone beam, em que os valores do ultrassom excederam as medidas da tomografia, porém de forma insignificante (32).

\section{Prevalência}

Manjunath et al. reuniram amostras de 336 indivíduos ( 186 homens e 150 mulheres) (33). A avaliação do fenótipo gengival foi através da transparência da sonda. No presente estudo, concluíram que o fenótipo espesso foi observado em 76,9\% dos indivíduos masculinos, e em mulheres foi de apenas 13,3\%. Já em relação ao fenótipo fino em homens foi visto 5,4\%, já em mulheres, 44,7\% (33).

Ocorreu um estudo, em 2018, por Alkan et al. com objetivo de avaliar o fenótipo gengival e a largura da gengiva queratinizada em dentes da região anterior de maxila em indivíduos com diferentes tipos de maloclusão (34). Um total de 181 pessoas foram estudadas, sendo I 8 mulheres e 63 homens. $\bigcirc$ grupo consistia em indivíduos com periodonto saudável e que não tinham sido submetidos a tratamentos ortodônticos. Estes foram divididos em grupos: classe I de Angle, classe II e classe III; cada grupo foi subdividido em subgrupos de acordo com a quantidade de apinhamento dentário na região anterior de maxila em: leve, moderado e grave. As medidas de dimensão da gengiva queratinizada foram feitas por uma sonda milimetrada, e a espessura gengival foi medida pelo método transgengival. $\bigcirc$ fenótipo era classificado de acordo com a espessura, sendo fenótipo fino (menor que I mm) e espesso (maior que I $\mathrm{mm}$ ). A prevalência do fenótipo gengival fino foi de $29,8 \%$, sendo mais comum no grupo classe II, grupo de apinhamento severo e em muIheres. A largura da gengiva queratinizada dos dentes 13 e 23 foi determinada como sendo mais estreita no grupo de apinhamento severo do que nos grupos de apinhamento leve e moderado. A relação entre a largura da gengiva queratinizada e a classificação de Angle não foi estatisticamente significativa (34).

Foi coletado por García Cortez et al., amostras de 550 indivíduos saudáveis sistemicamente (35). $\bigcirc$ parâmetro para avaliação do fenótipo foi da sobreposição da sonda milimetrada. Foi classificado como fino quando a gengiva apresentava translucidez nos 6 dentes avaliados; espesso quando não havia translucidez da sonda em 6 dentes; e misto quando não havia um padrão. Mulheres representavam 60,2\% do fenótipo fino ( $\mathrm{I}$ l). A idade, o tabagismo, os hábitos de higiene, a alimentação e a má oclusão não tiveram impacto significativo. Indivíduos com dentes ovais e triangulares apresentavam fenótipo fino (73\%), já os com dentes quadrados apresentavam fenótipo espesso (39,4\%), especialmente em homens (52,4\%) (35).

Em um estudo de 2020, foi feita por Rathod et al. uma coleta de 110 indivíduos (57 homens e 53 mulheres), sendo esses divididos em dois grupos, de acordo com sua má oclusão, e em 
seguida divididos conforme seu nível de apinhamento dentário (36). Foram avaliados os dentes anteriores da área estética, determinando por meio da transparência da sonda periodontal o fenótipo gengival e a largura da gengiva inserida pelo método de coloração histoquímica. $\bigcirc$ estudo teve como resultado a ausência de relação significativa entre o fenótipo gengival e a má oclusão de acordo com a classificação de Angle (36).

\section{DISCUSSÃO}

Em relação à prevalência do fenótipo periodontal ao gênero, foi demostrado em um estudo de Manjunath et al. que o fenótipo espesso foi observado em 76,9\% dos indivíduos masculinos, em mulheres foi de apenas 13,3\%. Já em relação ao fenótipo fino em homens foi visto 5,4\%, e em mulheres $44,7 \%$ (33). Discordando com as evidências relatadas, em um estudo de Amid et al. os fenótipos finos e espessos nos dentes anteriores superiores não mostraram parâmetros diferentes entre homens e mulheres (24). Em estudo de Alkan et al., constatou-se que o fenótipo gengival fino foi $29,8 \%$ mais comum no grupo classe II de Angle, grupo de apinhamento severo e em mulheres (34). Desarmonizando com tal achado, em 2020, Rathod et al. tiveram como conclusão não mostrar relação significativa entre o fenótipo gengival e a má oclusão de acordo com a classificação de Angle (36).

Continuando a abordagem sobre a prevalência, dessa vez comentando sobre outros fatores além do gênero, García Cortez et al. (35), em 2019, notaram em sua pesquisa que idade, tabagismo, hábitos de higiene, alimentação e má oclusão não tiveram impacto significativo na associação com o fenótipo gengival. Foi notada uma correlação entre o fenótipo e a morfologia dentária. Portanto, a quantidade de tempo que o paciente possui o hábito do tabagismo influencia bastante no aumento da espessura gengival. (35).

Abordando alguns achados clínicos importantes sobre o fenótipo periodontal, Maroso et al. concretizaram uma correlação entre a espessura gengival e o aparecimento de recessões (25). Yuan et al. descobriram uma ligação entre a espessura óssea e a espessura gengival, largura da coroa / comprimento da coroa, largura da gengiva queratinizada e curvatura da margem gengival livre (27). Discordando com os dados levantados, Karakis et al. não encontraram diferenças estatisticamente significativas nos parâmetros clínicos em pacientes com fenótipo espesso e fino, que foram submetidos à cirurgia de enxerto gengival livre (26).

Debatendo sobre as formas de diagnóstico do fenótipo periodontal, segundo Alves et al., em 2018, o método de TCCB para classificar a espessura e largura do tecido gengival apresenta valores aceitáveis de confiabilidade e pode ser considerado útil clinicamente (9). No estudo elaborado por Gkogkos et al. fez-se uma comparação entre a avaliação pelo ultrassom e a tomografia computadorizada de feixe cônico, em que os valores do ultrassom excederam as medidas da tomografia, porém de forma insignificante, o que indica a confiabilidade do método da determinação da espessura gengival (32). Porém, apesar da alta credibilidade no uso da tomografia, o Workshop Mundial para a Classificação das Doenças e Condições Periodontais e Peri-Implantes de 2017 preconizou a utilização do método de transparência gengival por meio da sonda periodontal (5). Em 20 I8, concluiu-se que profissionais com mais de 10 anos de atuação na área, independendo da especialização, tiveram mais facilidade em diagnosticar de forma correta o fenótipo gengival (3I).

\section{CONCLUSÃO}

Com o passar dos anos, muitos autores adotaram formas diferentes de classificação do fenótipo periodontal. Recentemente, no Workshop Mundial para a Classificação das Doenças e Condições Periodontais e Peri-Implantares, de 2017, foi uniformizada uma única nomenclatura: fenótipo periodontal. Sua classificação mais usual prevalece entre os termos "fino" e "espesso". Pode-se concluir que há uma grande prevalência do fenótipo fino no gênero feminino, e do espesso no gênero masculino. De acordo com os achados do presente estudo, o método de transparência da sonda milimetrada apresentou resultados satisfatórios, porém ainda faltam evidências científicas concretas a respeito do tema, principalmente para auxiliar os profissionais na conduta e plano de tratamento no seu dia a dia clínico.

Os autores declaram que não há conflito de interesse. 


\section{Autora de correspondência:}

Marcela Melo dos Santos

Estrada do Camorim 1003/306 - Rio de Janeiro, RJ, Brasil. marcela.santos@unigranrio.edu.br

\section{REFERÊNCIAS BIBLIOGRÁFICAS}

I. Cunha TD, Salgado IO, Costa LC, Galdino TM, Salgado

C. Proporção Áurea Em Dentes Permanentes Anteriores Superiores. Rev Interdisciplinar de Estudos Experimentais. 20।3;5:33-8.

2. Morley J, Eubank J. Macroesthetic elements of smile design. J Am Dent Assoc. 200 I; I 32:39-45.

3. Obradovic-Djuricic, Kostic L, Martinovic Z. Gingival and dental parameters in evaluation of esthetic characteristics of fixed restorations. Srp Arh Celok Lek. 2005; I 33(3-4): I 80-7.

4. Kao RT, Pasquinelli K.ThickVersus Thin Gingival Tissue: A Key Determinant in Tissue Response to Disease and Restorative Treatment. J of the California Dent Assoc. 2002;30(7):521-5.

5. Jepsen S, Caton JG, Albandar JM, et al. Periodontal manifestations of systemic diseases and developmental and acquired conditions: consensus report of workgroup 3 of the 2017 world workshop on the classification of periodontal and peri-implant diseases and conditions. J Periodontol. 2018;89:237-48.

6. Kao RT, Fagan MC, Conte G]. Thick vs thin gingival biotypes: a key determinant in treatment planning for dental implants. J of the California Dent Assoc. 2008;36: 193-8.

7. Lima LA. Estética em periodontia: quais os fatores que limitam a possibilidade dos resultados? em: Lotufo RFM, Lascala Jr NT. Periodontia e Implantodontia: desmistificando a ciência. 2003; Cap. 15.

8. Ronay $\mathrm{V}$, Sahrmann P, Bindl A, Attin T, Schmidlin PR. Current status and perspectives of mucogingival soft tissue measurement methods. J Esthet Restor Dent. 201 I;23: I 46-56.

9. Alves PHM, Alves TCLP, Pegoraro TA, Costa YM, Bonfante EA, de Almeida ALPF. Measurement properties of gingival biotype evaluation methods. Clin Implant Dent Relat Res. 2018; 00:1-5

10. Harris RJ. A comparative study of root coverage obtained with guided tissue regeneration utilizing a bioabsorbable membrane versus the connective tissue with partial-thickness double pedicle graft. J Periodontol. 1997;68:779-790.

I I. Kan JY, Rungcharassaeng K, Umezu K, Kois JC. Dimensions of periimplant mucosa: an evaluation of maxillary anterior single implants in humans. J Periodontol. 2003;74:557-562.

12. Müller HP, Heinecke A, Schaller N, Eger T. Masticatory mucosa in subjects with different periodontal phenotypes. J Clin Periodontol. 2000;27:621-6.

13. Müller HP, EgerT. Gingival phenotypes in young male adults.
J Clin Periodontol. 1997;24:65-7I.

14. Januário AL, Barriviera M, Duarte WR. Soft tissue conebeam computed tomography: a novel method for the measurement of gingival tissue and the dimensions of the dentogingival unit. J Esthet Restor Dent. 2008;20:366-73.

15. De Rouck T, Eghbali R, Collys K, et al. The gingival biotype revisited: transparency of the periodontal probe through the gingival margin as a method to discriminate thin from thick gingiva. J Clin Periodontol. 2009;36:428-33.

I6. Eghbali A, De Rouck T, De Bruyn H, Cosyn J. The gingival biotype assessed by experienced and inexperienced clinicians. J Clin Periodontol. 2009;36:958-63.

17. Kan JY, Marimoto T, Rungcharassaeng K, Roe P, Smith DH. Gingival biotype assessment in the esthetic zone: visual versus direct measurement. Int J Periodontics Restorative Dent. 2010;30:237-243.

I8. Fu JH, Yeh CY, Chan HL, Tatarakis N, Leong DJM, Wang $\mathrm{HL}$. Tissue biotype and its relation to the underlying bone morphology. J Periodontol. 20 I0;81:569-74.

19. Zweers J, Thomas RZ, Slot DE, Weisgold AS, van der Weijden FGA. Characteristics of periodontal biotype, its dimensions, associations and prevalence: a systematic review. J Clin Periodontol. 2014:41:958-71.

20. Cook DR, Mealey BL,Verrett RG, et al. Relationship between clinical periodontal biotype and labial plate thickness: an in vivo study. Int J Periodontics Restorative Dent. 20 I I;31:345-54.

21. Kim DM, Bassir SH, Nguyen TT. Effect of gingival phenotype on the maintenance of periodontal health: an American Academy of periodontology best evidence review. J Periodontol. 2020;91: 31 I-38.

22. "Phenotype." Merriam-Webster.com Dictionary, MerriamWebster, disponível em: https://www.merriam-webster.com/ dictionary/phenotype. Acessado em 28 de Fevereiro de 2021. 23. Lindhe J. Tratado de Periodontia Clínica e Implantodontia Oral. 4a ed. Rio de Janeiro : Guanabara Koogan; 2005.

24. Amid R, Mirakhori M, Safi Y, Kadkhodazadeh M, Namdari M. Assessment of gingival biotype and facial hard/soft tissue dimensions in the maxillary anterior teeth region using cone beam computed tomography. Arch Oral Biol. 20 I 7 Jul;79: I -6. 25. Maroso FB, Gaio EJ, Rösing CK, Fernandes MI. Correlation between gingival thickness and gingival recession in humans. Acta Odontol Latinoam. 20 I5;28(2): 162-6.

26. Karakış Akcan S, Güler B, Hatipoğlu H.The effect of different gingival phenotypes on dimensional stability of free gingival graft: A comparative 6-month clinical study. J Periodontol. 2019 Jul;90(7):709- I 7.

27.Yuan J, Guo QQ, Li Q, Sui YJ, Jiang BQ. Relationships among the periodontal biotype characteristics in the maxillary anterior. Hua Xi Kou Qiang Yi Xue Za Zhi. 2020 Aug I;38(4):398-403. 28. Yin $X J$, Wei BY, Ke XP, Zhang T, Jiang MY, Luo XY, et al. Correlation between clinical parameters of crown and gingival 
morphology of anterior teeth and periodontal biotypes. BMC Oral Health. 2020 Feb 19;20( I):59.

29. Egreja AM, Kahn S, Barceleiro M, Bittencourt S. Relationship between the width of the zone of keratinized tissue and thickness of gingival tissue in the anterior maxilla. Int J Periodontics Restorative Dent. 2012 Oct;32(5):573-9.

30. Müller HP, Schaller N, Eger T. Ultrasonic determination of thickness of masticatory mucosa: a methodologic study. Oral Surg Oral Med Oral Pathol Oral Radiol Endod. 1999 Aug;88(2):248-53.

31. Araújo LNM, Borges SB, Medeiros I,Amorim ACM, Barbosa CV, Gurgel BCV. Determinação do biótipo periodontal através da análise de fotografias intra-orais. Rev. odontol. UNESP. 2018 Oct [cited 2021 Feb 28]; 47(5):282-90.

32. Gkogkos A, Kloukos D, Koukos G, Liapis G, Sculean A, Katsaros C. Clinical and Radiographic Gingival Thickness Assessment at Mandibular Incisors: an Ex Vivo Study. Oral Health Prev Dent. 2020 Jun 8; I8(I):607-17.
33. Manjunath RG, Rana A, Sarkar A. Gingival Biotype Assessment in a Healthy Periodontium: Transgingival Probing Method. J Clin Diagn Res. 2015 May;9(5):ZC66-9.

34. Alkan Ö, Kaya Y, Alkan EA, Keskin S, Cochran DL. Assessment of Gingival Biotype and Keratinized Gingival Width of Maxillary Anterior Region in Individuals with Different Types of Malocclusion. Turk J Orthod. 2018 Mar;3 I ( I): I 3-20.

35. García-Cortés JO, Loyola-Rodríguez JP, Monárrez-Espino J. Gingival biotypes in Mexican students aged 17-19 years old and their associated anatomic structures, socio-demographic and dietary factors. J Oral Sci. 2019.6I (I): I56-63.

36. Rathod SR, Gonde NP, Kolte AP, Bawankar PV. Quantitative analysis of gingival phenotype in different types of malocclusion in the anterior esthetic zone. J Indian Soc Periodontol. 2020 Sep-Oct;24(5):4|4-20. 